\title{
Determination of optimal chevron configurations in surface-stabilized smectic $C^{\star}$
}

\author{
N.G. Migranov ${ }^{1}$, A.A. Kudreyko ${ }^{2, \dagger}$, D.N. Migranova ${ }^{1}$ \\ †akudreyko@rusoil.net \\ ${ }^{1}$ Bashkir State Pedagogical University, 3A Oct. Revilutsii St., 450000 Ufa, Russia \\ ${ }^{2}$ Ufa State Petroleum Technological University, 1 Kosmonavtov St., 450062 Ufa, Russia
}

\begin{abstract}
Configuration properties of layer orientation in smectic $\mathrm{C}^{*}$ type ferroelectric liquid crystals are theoretically described for constant temperature. The model proposed admits both symmetric and asymmetric configurations of smectic layers [chevron structures] in surface-stabilized liquid crystal cells, which [chevron structures] perturb ordering of smectic $\mathrm{C}^{\star}$ molecules in the so-called bookshelf geometry. The considered model reasonably disregards mass transfer between the smectic molecular layers. Since the length of smectic layers is permanent for any coordinate of the chevron tip, because of the fixed polar angle $\theta$, the locus of the chevron tip can be represented by ellipse. By using the continuum approach, the functional of the free energy density with the quadratic term of the electric field density is found. To determine energetically favorable structures of molecular layers, the Euler-Lagrange equation was solved, which is the dependence of the director's azimuthal angle $\varphi$-from the direction normal to the cell substrates. It is shown that only symmetric chevron structures exhibit minimum of free energy in the absence as well as in the presence of the electric field. This effect is caused by splay deformation of smectic layers. The stability analysis of the Euler-Lagrange equation for typical values of electric field and smectic $\mathrm{C}^{\star}$ parameters shows that the theoretical results are in agreement with the experimental data. The approach proposed for studying of the chevron configurations can explain the abundance of textures in smectic $C^{\star}$ liquid crystal cells.
\end{abstract}

Keywords: ferroelectric liquid crystals, chevrons, thin films, free energy density.

\section{Определение оптимальных конфигураций шевронов в поверхностно-стабилизированном смектике $\mathrm{C}^{*}$}

\author{
Мигранов Н.Г. ${ }^{1}$, Кудрейко А.А. ${ }^{2, \dagger}$, Мигранова Д.Н. ${ }^{1}$ \\ †akudreyko@rusoil.net
}

${ }^{1}$ Башкирский государственный педагогический университет, ул. Октябрьской революции 3А, 450000 Уфа

${ }^{2}$ Уфимский государственный нефтяной технический университет, ул. Космонавтов 1, 450062 Уфа

\begin{abstract}
Проведено теоретическое описание конфигурационных свойств слоёв сегнетоэлектрических жидких кристаллов типа смектик $\mathrm{C}^{\star}$ при постоянной температуре. Предлагаемая в этом письме модель допускает симметричные и несимметричные формы смектических слоёв [шевронные структуры] в поверхностно-стабилизированных жидкокристаллических ячейках, которые нарушают геометрию упаковки молекул - так называемой «книжной полки». Рассматриваемая модель обоснованно не учитывает массоперенос вещества между молекулярными слоями смектика С*. Поскольку длина смектических слоёв при изменении положении излома остаётся постоянной ввиду фиксированного значения полярного угла $\theta$, то в рассматриваемой модели геометрическое место точек излома смектических слоёв представляет собой эллипс. С помощью континуального подхода, получен функционал плотности свободной энергии системы с учётом квадратичного слагаемого, связанного с энергией электрического поля. Нахождение энергетически выгодных структур молекулярных слоёв производится с помощью решения уравнения Эйлера-Лагранжа - зависимости азимутального угла директора $\varphi$ от направления, перпендикулярного подложкам ячейки. Показано, что только симметричная структура шеврона обеспечивает минимум свободной энергии слоя смектика $\mathrm{C}^{*}[$ как при включённом так и выключенном электрическом поле]. Причиной такого поведения свободной энергии является сплей-деформация смектических слоёв. Анализ стабильности решения уравнения Лагранжа для диапазона допустимых значений электрических полей и параметров смектика $\mathrm{C}^{\star}$ показал, что теоретические результаты хорошо согласуются с экспериментальными данными. Предложенный подход к изучению конфигураций шевронов может объяснить изобилие разных текстур, которые наблюдаются в жидкокристаллических ячейках, содержащих смектик $\mathrm{C}^{\star}$.
\end{abstract}

Ключевые слова: сегнетоэлектрические жидкие кристаллы, шевроны, тонкие плёнки, плотность свободной энергии. 


\section{1. Введение}

Открытие Р. Мейером сегнетоэлектрических жидких кристаллов позволило развить новые направления физики конденсированного состояния. Известно, что сегнетоэлектрические жидкие кристаллы (СЖК) типа смектик $\mathrm{C}^{\star}\left(\mathrm{SmC}^{*}\right)$ являются геликоидальными. Эта геликоидальность может быть подавлена жёсткими граничными условиями или приложенным внешним электрическим полем, лежащим в плоскости смектических слоёв. Второе условие подавления связано с суммарным дипольным моментом единицы объёма [вектор поляризации $\mathbf{P}$ ], который в так называемых поверхностно-стабилизированных ячейках имеет ненулевое значение. Поэтому, молекулы SmC^ высокочувствительны к воздействию электрического поля. Сегнетоэлектрические жидкие кристаллы, имеющие очень малые времена отклика по сравнению с нематическими, являются на сегодня достаточно перспективными материалами для создания жидкокристаллических дисплеев нового поколения и оборудования для стекловолоконной оптики. Одной из главных проблем, которая препятствует быстрому внедрению СЖК в электрооптические устройства, является точная передача полутонов и высокая чувствительность даже к небольшим механическим воздействиям, в виде тряски, слабых ударов вследствие которого нарушается структура молекулярного упорядочивания и появляются дислокации. Причиной проблемы передачи полутонов является образование шевронов в жидкокристаллической ячейке.

К настоящему времени опубликовано немало работ, которые предлагают решения технических сложностей и объясняют физику взаимодействия шевронных структур с электрическим полем. Было показано, что формирование шевронных структур более энергетически выгодно по сравнению с так называемой «полочной» геометрией упаковки молекул $\mathrm{SmC}^{\star}[1,2]$. В работах [3-5] предлагаемые математические модели формирования шеврона представляли собой конкуренцию двух процессов: термического изменения толщины слоя $\mathrm{SmC}^{*}$ и эффекта памяти поверхности, которая сопротивлялась скольжению слоёв вдоль подложки. Поскольку слои $\mathrm{SmC}^{*}$ упругие и сцеплены с подложкой, то они объёмно деформируются в V-образные шевронные структуры.

Несмотря на большое внимание к шевронным структурам, до сих пор нет чёткого обоснования принципов их организации вне массопереноса. В настоящей работе обосновывается и рассматривается модель монослоя шевронной структуры, у которой геометрическое место точек излома - эллипс. Показано, что минимум свободной энергии соответствует симметричным шевронным слоям $\mathrm{SmC}^{\star}$. Этот результат согласуется с экспериментальными данными тем, что ассиметричные шевронные слои встречаются гораздо реже, чем симметричные. Расчеты проводятся для ячейки с жёсткими граничными условиями на подложках.

\section{2. Постановка задачи и основные уравнения}

Рассмотрим модель электрооптической ячейки жидкого кристалла типа $\mathrm{SmC}^{*}$ толщиной $d$, которая является поверхностно-стабилизированной, и находится в шевронной конфигурации. Пусть эта ячейка находится вдали от температуры фазового перехода $\mathrm{SmC}^{\star}-\mathrm{SmA}^{*}$ [6]. Это означает, что мы не можем пренебрегать малостью угла наклона директора $\mathbf{n}$ от единичного вектора нормали $\boldsymbol{a}$ смектического слоя. Для описания ориентации директора и структуры шевронного слоя, введем угол наклона слоев $\delta$ относительно плоскости $z=0$. Легко понять, что угол $\delta$ связан с полем смещения слоёв $u(x)$ соотношением $\operatorname{tg} \delta=-d u / d x$. Тогда для компонент директора - главной оптической оси слоя $\mathrm{SmC}^{*}$, получим

$$
\begin{gathered}
\mathbf{n}=(-\sin \theta \cos \delta \sin \delta+\sin \delta \cos \theta, \\
\sin \theta \cos \varphi, \sin \delta \sin \theta \sin \varphi+\cos \delta \cos \theta),
\end{gathered}
$$

где $\theta$ - угол наклона директора, отсчитываемый от нормали $\boldsymbol{a}$ к слою [полярный угол], а $\varphi-$ азимутальный угол, описывающий поворот директора вокруг вектора нормали, отсчитываемый от оси $y$ (Рис. 1). С другой стороны, $\varphi$ - это угол между осью $y$ и единичной проекцией вектора $\mathbf{n}$ на плоскость смектического слоя (с-директор). В рамках рассматриваемой модели мы будем полагать, что геометрическое место точек излома смектических слоёв - эллипс, фокусы которого расположены в точ-

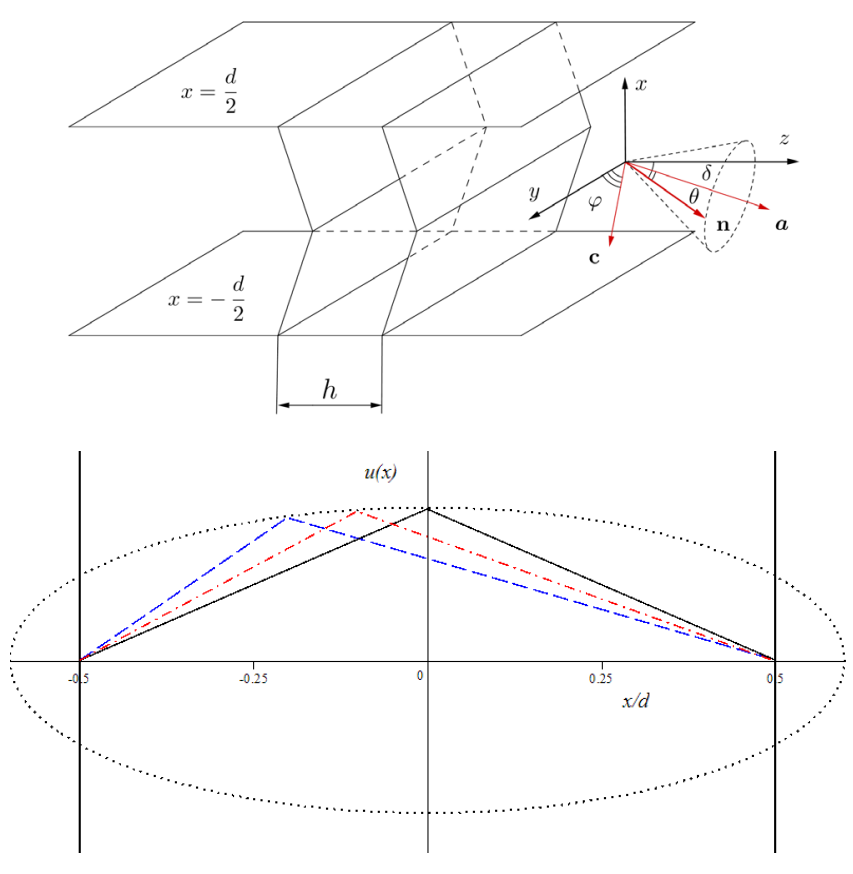

Рис. 1. Пространственная ориентация векторов $\boldsymbol{a}, \mathbf{c}$ и директора n в поверхностно-стабилизированном СЖК. Ось $x$ направлена перпендикулярно подложкам, ось $y$ направлена параллельно подложкам и излому шеврона (а). Модели полей смещения слоёв $\mathrm{SmC}^{\star}$, измеряемые вдоль оси $z$. Сплошные вертикальные линии - границы ячейки (b).

Fig. 1. Spatial orientation of vectors $\boldsymbol{a}, \mathbf{c}$ and director $\mathbf{n}$ in surfacestabilized ferroelectric liquid crystal. The $x$-axis is perpendicular the plates, the $y$-axis is parallel the plates and the chevron tip (a) Models of displacement fields in $\mathrm{SmC}^{\star}$ layers measured along the $z$-axis. The cell boundaries are shown by solid vertical lines (b). 
ках $x= \pm d / 2$. Это связано с тем, что длина смектического слоя при заданной температуре, вследствие постоянного наклона [величина угла зависит $\theta$ от температуры] директора в слое $\mathrm{SmC}^{\star}$ остаётся постоянной. Ассиметричные шевронные структуры были ранее рассмотрены в работах $[7,8]$. Выбор такой модели связан также и с тем обстоятельством, что мы рассматриваем молекулярный слой $\mathrm{SmC}^{\star}$ с жестким сцеплением с подложками вне массопереноса вещества при изменении конфигурации шеврона. Такое предположение допустимо, поскольку поведение слоёв $\mathrm{SmC}^{\star}$ в моменты времени после включения или выключения электрического полей находится вне нашей модели. Поэтому, такое расположение точек излома слоёв в плоскости $x z$ является единственно возможным.

Для определения оптимальной шевронной конфигурации, запишем плотность свободной энергии $f$, которая включает в себя плотность энергии упругости, связанной с искажением поля директора $f_{\text {elas }}$, деформацию смектических слоёв $f_{u}$ и плотность энергии взаимодействия молекул с электрическим полем $f_{\text {elec }}$. В рамках рассматриваемой модели, в одноконстантном приближении, представленные компоненты плотности свободной энергии имеют вид

$$
\begin{gathered}
f_{\text {elas }}=\frac{K}{2}\left((\nabla \cdot \mathbf{n})^{2}+(\nabla \times \mathbf{n})^{2}\right) \\
f_{u}=\frac{K_{u}}{2}\left(\frac{1}{\cos ^{2} \delta} \frac{d \delta}{d x}\right)^{2}+\frac{B}{2}\left(1-\frac{\cos \theta}{\cos \delta}\right)^{2} \\
f_{\text {elec }}=-(\mathbf{P} \cdot \mathbf{E})-\frac{1}{2}(\mathbf{D} \cdot \mathbf{E})
\end{gathered}
$$

где $K$ - усреднённый модуль упругости Франка, $K_{u}-$ модуль упругости Франка, связанный со сплей-деформацей смектических слоёв и $B-$ модуль сжатия смектических слоёв. Первое слагаемое в функционале (4) - плотность энергии спонтанной поляризации; второе слагаемое плотность энергии поляризации, возникшая благодаря образованию индуцированных диполей. Подробное исследование вектора спонтанной поляризации $\mathbf{P}$ и вектора электрического смещения D в хиральных смектиках представлено в ряде известных работ, например, $[9,10]$. Поскольку шевронные структуры представляют собой пересекающиеся плоскости, то в выражении плотности свободной энергии $f$ слагаемые, содержащие производную $d \delta / d x$ обратятся в ноль, а функционал $f$ примет вид:

$$
\begin{aligned}
f= & \frac{K}{2} \sin ^{2} \theta\left(\frac{d \varphi}{d x}\right)^{2}-P_{0} E \cos \delta \cos \varphi- \\
& -\frac{\varepsilon_{0} \varepsilon_{a} E^{2}}{2}(\cos \delta \sin \varphi \sin \theta-\cos \theta \sin \delta)^{2}- \\
& -\frac{\varepsilon_{0} \varepsilon_{\perp} E^{2}}{2}+\frac{B}{2}\left(1-\frac{\cos \theta}{\cos \delta}\right)^{2},
\end{aligned}
$$

где $\varepsilon_{0}-$ диэлектрическая постоянная, $P_{0}-$ спонтанная поляризация, $\varepsilon_{a}-$ диэлектрическая анизотропия и $\varepsilon_{\perp}-$ диэлектрическая проницаемость, перпендикулярная направлению преимущественной ориентации молекул [полю директора]. Для (5) легко получить уравнение
Эйлера-Лагранжа, решение которого определит пространственную ориентацию $c$-директора

$K \sin ^{2} \theta \frac{d^{2} \varphi}{d x^{2}}+P_{0} E \sin \varphi \cos \delta+$

$+\frac{\varepsilon_{0} \varepsilon_{a} E^{2}}{2}\left(\sin ^{2} \theta \cos ^{2} \delta \varphi \sin 2 \varphi-\frac{1}{2} \sin 2 \theta \sin 2 \delta \cos \varphi\right)$.

Из условия непрерывности поля директора $\mathbf{n}$ следует, что на линии излома смектических слоёв он должен лежать в плоскости подложек ячейки. Зная углы наклона смектических слоёв, слева $\delta_{1}$ и справа $\delta_{2}$ от плоскости излома (Рис. $1 \mathrm{~b}$ ), запишем следующие граничные условия [11] для так называемого $U$-состояния директора

$$
\begin{gathered}
\left.\varphi(0)\right|_{x \rightarrow-\xi}=-\varphi_{01},\left.\quad \varphi(0)\right|_{x \rightarrow+\xi}=\varphi_{02}, \\
\varphi\left( \pm \frac{d}{2}\right)= \pm \frac{\pi}{2},
\end{gathered}
$$

где $\varphi_{01}=\arcsin \left(\operatorname{tg} \delta_{1} / \operatorname{tg} \theta\right)$ и $\varphi_{02}=\arcsin \left(\operatorname{tg} \delta_{2} / \operatorname{tg} \theta\right)-$ граничные условия азимутальных углов слева и справа от плоскости $y z$. Подробное описание и физический смысл $D$ - и $U$-состояний директора представлен во многих работах, напр., [3]. В нашей работе мы обратим внимание на конфигурации шевронного $\mathrm{SmC}^{\star}$, которые обеспечивают минимум функционала (5).

\section{3. Оптимальные конфигура- ции шевронной структуры}

\section{1. Электрическое поле выключено}

В отсутствии электрического поля, решение уравнения (6) с граничными условиями (7) имеет вид

$$
\varphi(x)=\left\{\begin{array}{lc}
\frac{\pi-2 \varphi_{01}}{d} x-\varphi_{01}, & -\frac{d}{2}<x \leq \xi ; \\
\frac{\pi-2 \varphi_{02}}{d} x+\varphi_{02}, & \xi<x \leq \frac{d}{2},
\end{array}\right.
$$

где $\xi$ является координатой линии излома смектического слоя. Подставив решение уравнения (6) - функцию $\varphi(x)$ в функционал $f$, получим выражение для энергии смектического слоя, приходящуюся на единицу площади

$$
\mathcal{F}=\int_{-d / 2}^{\xi} f(x) d x+\int_{\xi}^{d / 2} f(x) d x,
$$

где $d=5$ мкм. Сопоставив значения $\mathcal{F}$ при $\xi=\{-0.2 d ;-0.1 d ; 0\}$, можно заключить, что свободная энергия минимальна, если слой $\mathrm{SmC}^{*}$ сломан в центре между подложками. Такая зависимость свободной энергии от положения линии излома слоя $\mathrm{SmC}^{\star}$ определяется влиянием так называемой сплей-деформацией молекул $\mathrm{SmC}^{\star}$. Более того, поскольку ассиметричные шевронные слои не могут сохранить целостность жёсткого сцепления с подложкой, то такая конфигурация менее устойчива. Элементарные расчёты показывают, что расхождение величины $\mathcal{F}$ между конфигурациями шевронов с изломами при $\xi=-0.2 d$ и $\xi=0$ составляет $\cong 19 \%$. Следовательно, устойчивыми будут симметричные шевронные конфигурации. 


\section{2. Электрическое поле включено}

При включённом электрическом поле $E$, направленном вдоль оси $x$, вектор спонтанной поляризации стремится занять положение вдоль силовых линий поля. Благодаря этому, молекулы разворачиваются по образующей конуса таким образом, что полярный угол $\theta$ остается неизменным, а азимутальный угол $\varphi$ изменяется в соответствии с заданными граничными условиями (7). Для расчётов будем использовать типичные значения SmC*: $K=5 \times 10^{-12} \mathrm{H}, B=4 \times 10^{6} \mathrm{H}, P_{0}=2.9 \times 10^{-4}$ Кл $/ \mathrm{M}^{2}$ и $\varepsilon_{a}=2.5$.

Численное решение уравнения (6) с граничными условиями (7) приводит к зависимостям $\varphi(x)$ при разных $\xi$, но для того, чтобы узнать какая функция $\varphi(x)$ обеспечивает минимум свободной энергии, необходимо сделать соответствующую подстановку в выражение (5), и рассчитать $\mathcal{F}$.

На Рис. 2 показаны три функции $\varphi(x)$ для возможных значений параметра $\xi$. Обратим внимание на то, что скачок азимутального угла $\varphi(x)$ при переходе с одной части плоскости слоя $\mathrm{SmC}^{\star}$ на другую обеспечивает непрерывное изменение директора. Расхождение величины $\mathcal{F}$ между конфигурациями шевронов с изломами при $\xi=-0.2 d$ и $\xi=0$ также составляет порядка $19 \%$.

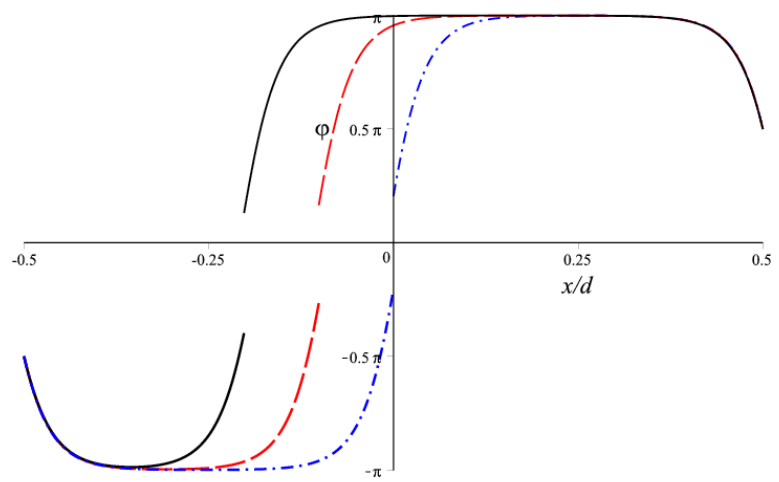

Рис. 2. Зависимость азимутального угла $\varphi$ от координаты $x$ для трёх возможных шевронных структур при $E=10^{5} \mathrm{~B} / \mathrm{M}$ : сплошная линия $-\xi=-0.2 d$; пунктирная линия $-\xi=-0.1 d$, штрих-пунктирная линия $-\xi=0$.

Fig. 2. The dependence of the azimuthal angle $\varphi$ versus the $x$-coordinate for three possible chevron structures when $E=10^{5}$ $\mathrm{V} / \mathrm{m}$. Here the solid line corresponds to $\xi=-0.2 d$; dashed line $\xi=-0.1 d$; dash-dotted line $-\xi=0$.
Полученный результат вполне согласуется с результатами рентгеновских исследований смектических слоёв $[10,12]$.

\section{4. Заключение}

В работе рассмотрена модель, согласно которой геометрическое место точек излома шевронной структуры эллипс. На основе континуального подхода, записан функционал плотности свободной энергии. Минимизация свободной энергии позволила определить поле директора для разных шевронных конфигураций. Численные расчёты показали, что минимум свободной энергии $\mathrm{SmC}^{\star}$ соответствует симметричным шевронным конфигурациям.

Благодарность/Acknowledgements. Работа авторского коллектива выполнена при поддержке грантов РФФИ № 16-32-00043 (А. А. К.) и № 14-02-97026 (Н. Г. М.).

\section{Литература/References}

1. N. Ul Islam, N. J. Mottram, S. J. Elston. Liquid Crystals. 26, 1059 (1999).

2. N. Vaupotic, S. Kralj, M. Copic, T. J. Sluckin. Phys. Rev. E. 54, 3783 (1996).

3. N. A. Clark, T. P. Rieker. Phys. Rev. A. 37 (3), 1053 (1988).

4. P. C. Willis, N. A. Clark, C. R. Safinya. Liquid Crystals. 11, 581 (1992).

5. J. Sabater, J. M. S. Pena, J. M. Otón. J. Appl. Phys. 773023 (1995).

6. A. D. Kiselev, V. G. Chigrinov, E. P. Pozhidaev. Phys. Rev. E. 75061706 (2007).

7. M. Oh-e, M. Isogai, T. Kitamura. Liquid Crystals. 11, 101 (1992).

8. R.E. Webster, N. J. Mottram, D. J. Cleaver. Phys. Rev. E. 68. 021706 (2003).

9. I.W. Stewart. The Static and Dynamic Continuum Theory of Liquid Crystals: A Mathematical Introduction, London, Taylor \& Francis (2004) 360 p.

10. S. T. Lagerwall. Ferroelectric and Antiferroelectric Liquid Crystals, Weinheim, Wiley-VCH (1999) 427 p.

11. V.P. Romanov, S. V. Ul'yanov, K. G. Chernyak, Physics of the Solid State. 52 (9) 1985 (2010).

12. J. C. Jones. Liquid Crystals. 42, 732 (2015). 